\title{
A false alarm of COVID-19 pneumonia in lung cancer: a case report of anti-PD-1 related pneumonitis and literature review
}

\section{Ying Dai}

The first affilliated hospital of Anhui Medical University

\section{Ying Dai}

The first affilliated hospital of Anhui Medical University

Sha Liu

The first affilliated hospital of Anhui Medical University

Sha Liu

The first affilliated hospital of Anhui Medical University

\section{Zhiyan Zhao}

The first affilliated hospital of Anhui Medical University

\section{Zhiyan Zhao}

The first affilliated hospital of Anhui Medical University

\section{Xiaqiu Li}

The first affilliated hospital of Anhui Medical University

\section{Xiaqiu Li}

The first affilliated hospital of Anhui Medical University

\section{Yiruo Zhang}

The first affilliated hospital of Anhui Medical University

\section{Yiruo Zhang}

The first affilliated hospital of Anhui Medical University

\section{Pingping Liu}

The first affilliated hospital of Anhui Medical University

\section{Pingping Liu}

The first affilliated hospital of Anhui Medical University

\section{Yingying Du (D303162554@qq.com )}

The first affilliated hospital of Anhui Medical University

\section{Yingying Du ( $\nabla 03162554 @ q q . c o m$ )}

The first affilliated hospital of Anhui Medical University

\section{Case Report}


Keywords: COVID-19, pneumonitis, immunotherapy

Posted Date: July 14th, 2020

DOI: https://doi.org/10.21203/rs.3.rs-41095/v1

License: (c) (1) This work is licensed under a Creative Commons Attribution 4.0 International License. Read Full License

Version of Record: A version of this preprint was published at Journal of Medical Case Reports on February 1st, 2021. See the published version at https://doi.org/10.1186/s13256-020-02619-y. 


\section{Abstract}

Background: The fatal toxicity of anti-PD-1/PD-L1 agents is pneumonitis. The diagnosis consists of the history of immunotherapy, clinical symptoms and presentation of computed tomography (CT) imaging. The typical CT findings include ground-glass opacities. Based on the similar radiographic feature with 2019 Novel Coronavirus (COVID-19) pneumonia, clinicians are cautious to evaluate diagnosis especially in COVID-19 epidemic areas. Case presentation: Herein we report a 67-year-old male patient with advanced non-small cell lung cancer developed pneumonitis post Sintilimab injection. The dyspnea appeared at the 15th day of close contact with his son who returned from Wuhan, but not accompanied with fever. The chest CT indicated peripherally subpleural lattice opacities at the inferior right lung lobe and bilateral thoracic infusion. The real-time reverse-transcription polymerase-chain-reaction (RT-PCR) from double swab samples within 72 hours remained negative. The patient was thereafter treated with prednisolone and antibiotics for over two weeks. Thereafter the chest CT demonstrated the former lesion almost absorbed, in line with prominently falling CRP level. The anti-PD-1 related pneumonitis with bacterial infection was diagnosed finally based on the clinical evidence and good response to the prednisolone and antibiotics.

Conclusion: Both ani-PD-1 related pneumonitis and COVID-19 pneumonia harbor the common clinical symptom and the varied features of CT imaging. Differential diagnosis was based on the epidemiological and immunotherapy histories, RT-PCR tests. The response to glucocorticoid can indirectly help the diagnosis.

\section{Background}

Immune checkpoint blockade monoclonal antibodies have revolutionized anti-tumor treatments in advanced lung cancer ${ }^{1}$. Among the unique toxicity due to the immunotherapy, pneumonitis is the severe and fatal immune-related adverse event (irAE) ${ }^{2}$, which is defined as noninfectious focal and diffuse inflammation of lung parenchyma ${ }^{3}$. The diagnosis was based on the clinical symptom and exclusion of pneumonia and other pulmonary infections including coronavirus disease 2019 (COVID-2019). The typical features of CT imaging of COVID-2019 are multifocal bilateral ground glass opacities (GGOs) with patchy consolidations, distributed peripherally in subpleural area of posterior part or lower lobes in lung. The diagnosis was fundamentally confirmed by positive real-time reverse-transcription-polymerase-chainreaction (RT-PCR) results by respiratory or blood samples. Herein we report a COVID-2019 suspect case of one advanced lung cancer patient present with pneumonitis post sintilimab injection. The negative RTPCR of coronavirus results and good response to prednisone has consolidate the diagnosis of anti-PD-1 related pneumonitis.

\section{Case Presentation}

A 67-year-old male smoker was present with nonproductive cough and breathless. The chest CT imaging showed central lung cancer located in left lobe, accompanied with pulmonary artery invasion, obstructive 
atelectasis and pleural effusion. The biopsy pathology from bronchoscopy indicated squamous cell carcinoma. He was treated with 10 cycles of sintilimab, concurrent with chemotherapy containing gemcitabine and carboplatin in the first 4 cycles. The immunotherapy was replaced by paclitaxel for one cycle when CT evaluation suggested progressed disease. The dyspnea appeared at the 15th day of close contact with his son who returned from Wuhan, but not accompanied with fever. The chest CT (Fig. 1A) indicated peripherally subpleural lattice opacities at the inferior right lung lobe and bilateral thoracic infusion. The complete blood count showed increased white blood cell (WBC) and neutrophilic granulocyte with concurrent decreased lymphocyte. The $C$ reaction protein (CRP) level was $97.68 \mathrm{mg} / \mathrm{L}$ with normal value of procalcitonin. As suspect of COVID-19 infection, the patient was treated in isolation ward and the double RT-PCR results from swab samples within 72 hours remained negative. No pathogen was found from sputum culture. The patient was thereafter treated the daily dose of $80 \mathrm{mg}$ prednisolone and meropenem for 7 days. At the third day post treatment, the chest CT (Fig. 1B) showed attenuated inflammatory lesion. The daily dose of prednisolone was stepwise reduced to $40 \mathrm{mg}$ for 7 days and then minimally $20 \mathrm{mg}$. Secondary to 7 days of piperacillin tazobactam injection, the chest CT (Fig. 1C) demonstrated the former lesion almost absorbed, in line with prominently falling CRP level to 22.17 $\mathrm{mg} / \mathrm{L}$. The anti-PD-1 related pneumonitis with bacterial infection was diagnosed finally based on the clinical evidence and good response to the prednisolone and antibiotics. Due to non-stopping hemoptysis throughout the disease, the patient started with afatinib and the stable disease was evaluated by CT imaging. He died post one month of oral treatment.

\section{Discussion And Conclusion}

Pneumonitis can develop at any time after initiation of immunotherapy. The diagnosis mainly relies on the CT images, infectious disease (ID) consult and pulmonary consult. The bronchoscopy is also alternative if required ${ }^{4}$. In CT imaging, the pneumonitis is more extensive in the lower lobes instead of middle and upper lobes. The radiographic features vary, but the majority displays GGOs in cryptogenic organizing pneumonia (COP) pattern. Given the similar changes by CT imaging, it is difficult to differentiate immunotherapy associated pneumonitis and COVID-19 pneumonia solely by CT assessment.

To diagnose COVID-2019, the positive real time RT-PCR assay result is fundamental. However, the first task of clinical diagnosis is to confirm Wuhan exposure history or close contact with people from Wuhan or COVID-19 patients in the latest two weeks according to China updated the novel coronavirus pneumonia diagnosis and treatment program (trial version) ${ }^{4,5}$. Other criteria include fever and/or respiratory symptoms, imaging evidence of viral pneumonia and normal or low white blood cell count or lymphopenia.

In our case, the hypoxemia firstly appeared at the 15th day of close contact with his son, who has residential history in Wuhan. The son was still suspicious of asymptomatic COVID-19 infection in spite of normal chest CT imaging and the complete blood cell count test, as his coronavirus status maintained unknown. The following negative RT-PCR tests from the patient and good response to prednisolone 
indirectly supported the diagnosis of anti-PD-1 related pneumonitis. Additionally, the son's RT-PCR tests in May was also negative, which consolidated the conclusion mentioned above.

Steroid therapy is still controversial in the management of COVID-19 in contrast to its vital role to treat anti-PD-1 related pneumonitis. In pandemic areas, the anti-PD-1 related pneumonitis was to some extent covered by coronavirus pneumonia due to the alert of COVID-19, leading to prolonged diagnosis and delayed treatment. Therefore, the routine screening of COVID-19 has been generally recommended before hospitalization in China. In conclusion, the differential diagnosis of both diseases should fundamentally base on epidemiology, RT-PCR tests and stepwise immunotherapy history.

\section{Abbreviations}

CT: computed tomography

CRP: $C$ reaction protein

COP: cryptogenic organizing pneumonia

COVID-19: 2019 Novel Coronavirus

irAE: immune-related adverse event

ID: infectious disease

GGOs: ground glass opacities

RT-PCR: the real-time reverse-transcription polymerase-chain-reaction

WBCIwhite blood cell

\section{Declarations}

\section{Ethics approval and consent to participate}

Not applicable

\section{Consent for publication}

Written informed consent for publication of their clinical details and/or clinical images was obtained from the patient/parent/guardian/ relative of the patient. A copy of the consent form is available for review by the Editor of this journal.

\section{Availability of data and materials}

All data generated or analyzed during this study are included in this published article. 


\section{Competing interest}

None to declare.

\section{Funding}

This work was supported by the grant from Anhui Key Research and Development Project (1704a0802163). The funders had no role in study design, data collection and analysis, decision to publish or preparation of the manuscript.

\section{Author's contribution}

YD and PPL drafted the manuscript, prepared the figures and performed the final revision of the manuscript. XQL, ZYZ, YRZ and SL extracted the clinical data from clinical records and prepared figures. the manuscript. YYD revised the manuscript for important intellectual content. All authors read and approved the final versions of the manuscript for submission.

\section{Acknowledgment}

The authors thank Prof. Hua Wang for editorial assistance.

\section{References}

1. Topalian SL, Hodi FS, Brahmer JR, et al. Safety, activity, and immune correlates of anti-PD-1 antibody in cancer. N Engl J Med 2012; 366: 2443-2454. 2012/06/05. DOI: 10.1056/NEJMoa1200690.

2. Naidoo J, Page DB, Li BT, et al. Toxicities of the anti-PD-1 and anti-PD-L1 immune checkpoint antibodies. Ann Oncol 2015; 26: 2375-2391. 2015/09/16. DOI: 10.1093/annonc/mdv383.

3. Balaji A, Verde F, Suresh K, et al. Pneumonitis From Anti-PD-1/ PD-L1 Therapy. Oncology (Williston Park) 2017; 31: 739-746, 754. 2017/10/31.

4. Bala-Hampton JE, Bazzell AF and Dains JE. Clinical Management of Pneumonitis in Patients Receiving Anti-PD-1/PD-L1 Therapy. J Adv Pract Oncol 2018; 9: 422-428. 2019/02/06.

5. Ge H, Wang X, Yuan X, et al. The epidemiology and clinical information about COVID-19. Eur J Clin Microbiol Infect Dis 2020; 39: 1011-1019. 2020/04/16. DOI: 10.1007/s10096-020-03874-z.

\section{Figures}



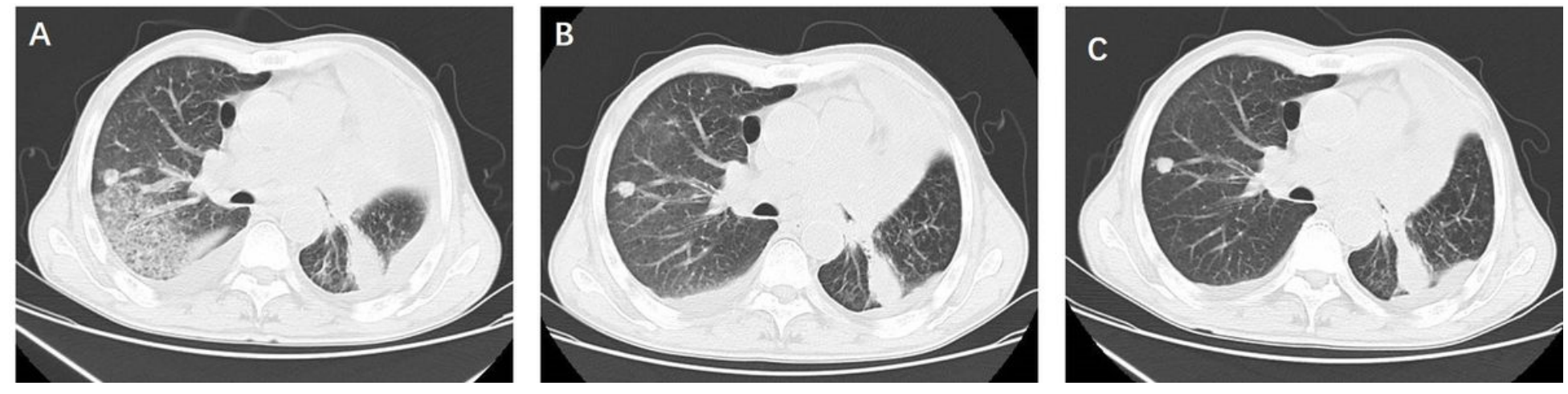

Figure 1

Assessment of pneumonitis lesion via chest CT scan before (A) and after 3 days (B) and 7 days (C) of prednisolone and antibiotics treatment. 